\title{
Article \\ Self-Perceived Physical Activity and Adherence to the Mediterranean Diet in Healthy Adolescents during COVID-19: Findings from the DIMENU Pilot Study
}

\author{
Angelo Galluccio 1,2, ${ }^{+}$, Giovanna Caparello 1,3, ${ }^{+}$, Ennio Avolio ${ }^{1,3}$, Emanuele Manes ${ }^{1}$, Simona Ferraro ${ }^{1,3}$, \\ Cinzia Giordano ${ }^{4,5}$, Diego Sisci ${ }^{4,5, \mp(1)}$ and Daniela Bonofiglio $4,5, *, \neq$ (D) \\ 1 Health Center srl, 87100 Cosenza, Italy; angelo.galluccio@yahoo.it (A.G.); \\ caparello.giovanna@gmail.com (G.C.); ennioavolio@libero.it (E.A.); emanuelemanes85@gmail.com (E.M.); \\ ferrarosimona@hotmail.it (S.F.) \\ 2 Department of Clinical and Experimental Medicine, University Magna Graecia, 88100 Catanzaro, Italy \\ 3 School of Specialization in Food Sciences, University of Rome Tor Vergata, 00133 Rome, Italy \\ 4 Department of Pharmacy, Health and Nutritional Sciences, University of Calabria, \\ 87036 Arcavacata di Rende, Italy; cinzia.giordano@unical.it (C.G.); diego.sisci@unical.it (D.S.) \\ 5 Centro Sanitario, University of Calabria, 87036 Arcavacata di Rende, Italy \\ * Correspondence: daniela.bonofiglio@unical.it; Tel.: +39-0984-496208; Fax: +39-0984-496203 \\ + These authors contributed equally to this work. \\ $\ddagger$ Senior joint authors.
}

Citation: Galluccio, A.; Caparello, G.; Avolio, E.; Manes, E.; Ferraro, S.; Giordano, C.; Sisci, D.; Bonofiglio, D. Self-Perceived Physical Activity and Adherence to the Mediterranean Diet in Healthy Adolescents during COVID-19: Findings from the DIMENU Pilot Study. Healthcare 2021, 9, 622. https://doi.org/10.3390/ healthcare 9060622

Academic Editors: Munjae Lee and Kyu-sung Lee

Received: 1 May 2021

Accepted: 21 May 2021

Published: 23 May 2021

Publisher's Note: MDPI stays neutral with regard to jurisdictional claims in published maps and institutional affiliations.

Copyright: (c) 2021 by the authors. Licensee MDPI, Basel, Switzerland. This article is an open access article distributed under the terms and conditions of the Creative Commons Attribution (CC BY) license (https:// creativecommons.org/licenses/by/ $4.0 /)$.

\begin{abstract}
The global pandemic coronavirus disease (COVID-19) resulted in restrictions which forced adolescents to stay at home and influenced their food habits and lifestyles with potential negative health impact. This study aims to investigate the self-reported physical activity (PA) and eating habits related to the consumption of Mediterranean foods in a sample of adolescents during the COVID-19 lockdown enrolled into the DIMENU study. A web survey was launched for 91 adolescents (aged 15-17 years) to assess their adherence to the Mediterranean Diet using the KIDMED test and lifestyle habits using a questionnaire designed following recommendations by Italian National Institute of Health (ISS score). Our results indicate that most of the sample declared no changes in eating habits and PA without sex differences. After dividing the sample into active and sedentary groups based on the self-perceived PA, we found that KIDMED and ISS scores were significantly higher $(p=0.0028$ and $p=0.0001$, respectively) in active adolescents. Moreover, KIDMED was positively correlated with ISS only in active adolescents $(r=0.311, p=0.0185)$. In conclusion, our data underline the impact of the PA on the Mediterranean diet adherence in adolescents during the lockdown, suggesting the usefulness of promoting wellness programs directed towards inactive individuals to increase their awareness on the importance of healthy lifestyles.
\end{abstract}

Keywords: physical activity; Mediterranean diet; lifestyle interventions; eating habits; adolescence

\section{Introduction}

On 30 January 2020, the World Health Organization (WHO) Emergency Committee declared a global health emergency based on growing case notification rates at Chinese and international locations caused by a novel coronavirus (COVID-19) [1]. After a few months, COVID-19 had been labeled a global pandemic by the WHO with a decree that provided for the adoption of preventive measures be taken to mitigate the viral spread [2]. In Italy, the procedures for containing the extension of COVID-19 have therefore been implemented with strong implications in the health, social, and economic fields. The public health measures imposed on citizens by the Italian Government include the obligation to practice a social distance (at least $2 \mathrm{~m}$ between individuals) and avoid social gatherings, limit contact with elderly individuals and people with poor health, avoid gestures such as handshakes, crowded places, and nonessential meetings [3]. Among these, there was 
the abrupt interruption of school programs for children and adolescents who by mandate had to remain in their homes during the lockdown aimed at containing and mitigating the spread of COVID-19. Thus, began the so-called "distance learning" in which teachers and students carried out digital teaching and distance learning from their own homes. The same closure also affected other institutional and commercial activities, such as gyms and various fitness and recreational centers. The consequences of these confinement measures led to increased sedentary behaviors, exposing the young and adult population to a greater risk of developing or worsening chronic health conditions [4-6]. For adolescents, physical activity (PA) is closely coupled to school-related activities [7]. Therefore, the closure of schools during the COVID-19 pandemic, which also compromises PA participation, caused the risk of longer-term sedentary behaviors. Emerging evidence suggests that PA levels during the pandemic declined in youth [8]. Such necessary restrictions, potentially compromising the lifestyle in terms of physical fitness, also contribute to impacts on eating habits and on maintaining a healthy and varied diet. Indeed, recent studies showed a higher prevalence of inactivity among adolescents during the lockdown, along with changes in number of meals/day and nutritional choices associated with a higher caloric intake [9-11]. This situation was particularly serious for maintaining a good state of health in adolescence, because the performance of an optimal level of physical exercise brings many benefits on the parameters of body composition, on the blood profile as well as lipid and carbohydrate metabolism [12]. Since diet is one of the main factors contributing to healthy status [13], it is conceivable that a situation in which the limited availability of food and of daily grocery shopping supply may lead to reduce the consumption of fresh foods in favor of highly processed ones rich in fats, sugars, and salt. Adolescents are in a vulnerable age group requiring careful consideration by caregivers to allow for maintaining health status during the lockdown. In the context of the global COVID-19 pandemic, is important to evaluate the effect of confinement on PA and dietary behaviors covering whole populations or age groups such as in adolescents. Investigating how PA and eating behaviors can be affected by lengthy restrictions is necessary to determine and develop appropriate recommendations in terms of lifestyle modifications for future lockdown policies. Therefore, the aim of this web survey was to examine self-reported physical activity levels and perceived active or sedentary behavior along with the assessment of eating habits related to the consumption of Mediterranean foods in a group of adolescents during the COVID-19 pandemic.

\section{Materials and Methods}

\subsection{Survey Development}

The present online research was developed within the trial DIMENU (Dieta Mediterranea e Nuoto-Mediterranean Diet and Swimming) funded by the EU Regional Operational Program Calabria, Italy (POR Calabria FESR-FSE 2014-2020) (prot.\#52243/2017) [12], in which a group of adolescents, aged 15 to 17 years, was recruited from students of the public high school "Istituto Istruzione Superiore"-Castrolibero (CS) and several swim, soccer, and squash teams located in Calabria Region, Italy before the COVID-19 pandemic period. This pilot study was carried out from March to May 2020 in 91 adolescents (42 females and 49 males) who received an invitation by e-mail explaining the purpose of the investigation and inviting recipients to click on the hypertext link to invoke the web browser, presenting the questionnaire. In this survey, $100 \%$ of the replies were received. This study was conducted according to the guidelines laid down in the Declaration of Helsinki and approved by the ethics committee of the University of Calabria, Italy (\#5727/2018). Written informed consent was obtained from all participants' parents.

\subsection{Questionnaire}

The questionnaire (DIMENU—Lifestyle Survey, DIMENU-LS) (Table A1 in Appendix A), including 30 questions, was designed to assess changes in both lifestyle and eating habit behaviors during the COVID-19 outbreak. The online questionnaire was divided into three different parts: (1) KIDMED test, used to assess the adherence to the Mediterranean 
diet (MD) [14], that was updated according to the new MD pyramid by the International Foundation of Mediterranean diet (IFMed) [15]. The MD adherence score, from 0 to 12, was based on a 16-point test. Questions denoting a positive aspect with respect to the MD were assigned a value of +1 (consumption of fruits, vegetables, fish, legumes, whole cereals or grain, nuts, oil, dairy products, and yogurt) and those with a negative connotation -1 (skipping breakfast, consumption of baked goods, sweets, and consumption of delivery junk foods). Level of adherence to MD was indicated as follows: high adherence to MD ( $\geq 8$ points), medium adherence to MD (4-7 points), low adherence to MD ( $\leq 3$ points). (2) ISS Score designed following recommendations from "Physical activity guidelines for children and young people" promoted by EpiCentro website belonging to the National Institute of Health (ISS) [16] to counter the increase of sedentary lifestyles and promoting an active lifestyle at home during the period of social isolation. It was used to assess active/sedentary lifestyle of the participants during the COVID-19 lockdown. The ISS score was based on a 7-point test, where from 0 to 3 points indicated a low score and from 4 to 7 points indicated a high score. (3) General questions on the actual perception of changes in lifestyle and eating habits at home. Once completed, each questionnaire was transmitted to the Google platform and the final database was downloaded as a Microsoft Excel sheet.

\subsection{Statistical Analysis}

Data were analyzed by SigmaPlot for Windows Version 12.0 (Systat, San Jose, CA, USA) and reported as mean, standard deviation (SD) and range. Data normality was verified by the Kolmogorov-Smirnov test (with Lilliefors' correction). The statistical differences between quantitative variables were evaluated by Student's $t$-test. Qualitative variables were described as frequencies (\%) and the statistical differences were evaluated by chi-squared test. The radar charts were built to display the percentage of participants currently adherent to each dietary/lifestyle recommendation respect to the ideal situation (100\% compliance). Relationship between variables was evaluated by multivariate linear regression and linear regression analyses. The association between variables is graphically represented through a straight line calculated using the regression coefficient. Pearson's correlation test was used to assess statistical significance. Statistical significance was set at $p<0.05$.

\section{Results}

\subsection{Description of Study Sample}

A total of 91 adolescents (aged 15-17 years, 42 females and 49 males), enrolled into the DIMENU study [12], completed the online questionnaire on physical activity behaviors, eating habits changes, eating frequency and adherence to the MD during the lockdown period. A detailed breakdown of the characteristics of the total sample population, divided into girls and boys, is given in Table 1.

Notably, a substantial proportion of participants currently considered their lifestyle active $(63 \%)$ with the highest ISS score in most of the total sample, which was independent of gender (high score: total sample: $78 \%$, females: $74 \%$, males: $82 \%$ ). With regards to eating habits during the COVID-19 lockdown, most of the population declared no changes (total sample: $63 \%$, females: $59 \%$, males: $65 \%$ ), while the percentages of total adolescents who consumed 4 and 5 meals / day were $39 \%$ and 26\%, respectively. The KIDMED questionnaire, assessing the compliance to the MD recommendations, revealed an average adherence score in total adolescents, again without gender differences, with approximately half of the total population declaring medium adherence to the MD (medium adherence: total sample: $49 \%$, female $48 \%$, male $51 \%$ ). 
Table 1. General characteristics of our study population.

\begin{tabular}{|c|c|c|c|}
\hline Characteristics & Total Sample & Girls & Boys \\
\hline Subjects (number) & 91 & $42(46 \%)$ & $49(54 \%)$ \\
\hline Age (years $\pm \mathrm{SD}$, range) & $16.60 \pm 1.28(13-19)$ & $16.83 \pm 1.27(14-18)$ & $16.40 \pm 1.26(13-19)$ \\
\hline \multicolumn{4}{|l|}{ Current lifestyle } \\
\hline Sedentary & $34(37 \%)$ & $18(20 \%)$ & $16(17 \%)$ \\
\hline Active & $57(63 \%)$ & $25(26 \%)$ & $32(37 \%)$ \\
\hline ISS score (M $\pm S D$, range) & $5.37 \pm 1.19(1-7)$ & $5.23 \pm 1.1(1-7)$ & $5.49 \pm 1.27(3-7)$ \\
\hline Low $(0-3)$ & $20(22 \%)$ & $11(26 \%)$ & $9(18 \%)$ \\
\hline High (4-7) & $71(78 \%)$ & $31(74 \%)$ & $40(82 \%)$ \\
\hline Eating habits change & $34(37 \%)$ & $17(41 \%)$ & $17(35 \%)$ \\
\hline \multicolumn{4}{|l|}{ Meals/day } \\
\hline 1-2 meals/day & $4(4 \%)$ & 0 & $4(8 \%)$ \\
\hline 3 meals/day & $28(31 \%)$ & $15(36 \%)$ & $13(27 \%)$ \\
\hline 4 meals/day & $35(39 \%)$ & $15(36 \%)$ & $20(41 \%)$ \\
\hline 5 meals/day & $24(26 \%)$ & $12(28 \%)$ & $12(24 \%)$ \\
\hline KIDMED score (M $\pm \mathrm{SD}$, range) & $6.52 \pm 2.45(0-11)$ & $6.28 \pm 2.68(1-11)$ & $6.73 \pm 2.24(0-11)$ \\
\hline Optimal adherence $(\geq 8)$ & $37(41 \%)$ & $16(38 \%)$ & $21(43 \%)$ \\
\hline Medium adherence $(4-7)$ & $45(49 \%)$ & $20(48 \%)$ & $25(51 \%)$ \\
\hline Poor adherence $(\leq 3)$ & $9(10 \%)$ & $6(14 \%)$ & $3(6 \%)$ \\
\hline
\end{tabular}

\subsection{Multiple Regression Analysis with KIDMED or Lifestyle and ISS, Age, and Sex}

Next, in multiple linear regression we analyzed the KIDMED score as well as lifestyle habits in relation to ISS score using age and sex as independent variables in the total sample. Interestingly, we observed that the KIDMED score can be predicted from a linear combination of the ISS score $(\beta=0.375, p<0.001)$, age $(\beta=0.140 p=0.170)$, and sex $(\beta=0.076 p=0.454)$ (Table 2$)$. Similarly, lifestyle can be predicted from a linear combination of the ISS score $(\beta=0.432, p<0.001)$, age $(\beta=-0.087 p=0.373)$, and $\operatorname{sex}(\beta=0.0463 p=0.642)$ (Table 2). These data suggest a direct relationship between ISS and both KIDMED and lifestyle, independent of age and sex.

Table 2. Multiple linear regression analysis between KIDMED as well as lifestyle habits and a set of different variables.

\begin{tabular}{cccccccc}
\hline \multirow{2}{*}{ Variables } & \multicolumn{3}{c}{ KIDMED } & \multicolumn{3}{c}{ Lifestyle Habits } \\
\cline { 2 - 8 } & $\boldsymbol{\beta}$ & $\boldsymbol{s e}$ & $\boldsymbol{p}$-Value & $\boldsymbol{\beta}$ & $\boldsymbol{s}$ e & $\boldsymbol{p}$-Value \\
\hline ISS & 0.375 & 0.206 & $<\mathbf{0 . 0 0 1}$ & 0.432 & 0.039 & 0.001 \\
\hline Age & 0.140 & 0.194 & 0.170 & -0.087 & 0.037 & 0.642 \\
\hline Sex & 0.076 & 0.491 & 0.454 & 0.045 & $\mathrm{R}^{2}=0.215$ & Adj $\mathrm{R}^{2}=0.188$ \\
\hline
\end{tabular}

For each variable, the linear regression coefficient $(\beta)$, the standard error (se), adjusted R-squared (Adj $\mathrm{R}^{2}$ ), and the statistical significance ( $p$-value) are reported.

\subsection{Dietary and Physical Activity Behaviors in Sedentary and Active Adolescents during the Lockdown Period}

Based on the self-reported PA behaviors during COVID-19-related restrictions, we divided our population in active and sedentary and compared the percentages of eating habit change, frequency of meals a day, and KIDMED and ISS scores between the two groups of adolescents (Table 3 ). 
Table 3. Dietary and physical activity habits in sedentary and active adolescents during the lockdown.

\begin{tabular}{|c|c|c|c|}
\hline \multirow{2}{*}{ Characteristics } & \multicolumn{2}{|c|}{ Total Sample $(n=91)$} & \multirow{2}{*}{$p$-Value } \\
\hline & Sedentary $(n=34)$ & Active $(n=57)$ & \\
\hline Age (years $\pm S D$, range) & $16.88 \pm 1.26(15-19)$ & $16.43 \pm 1.28(13-19)$ & 0.1063 \\
\hline ISS score (M $\pm S D$, range) & $4.67 \pm 1.22(1-7)$ & $5.78 \pm 1.2(3-7)$ & 0.0001 \\
\hline Low $(0-3)$ & $6(18 \%)$ & $1(2 \%)$ & 0.005 \\
\hline High (4-7) & $28(82 \%)$ & $56(98 \%)$ & 0.005 \\
\hline Eating habits change & $16(47 \%)$ & $18(31 \%)$ & 0.1398 \\
\hline \multicolumn{4}{|l|}{ Meals/day } \\
\hline 1-2 meals/day & $2(6 \%)$ & $2(4 \%)$ & 0.5931 \\
\hline 3 meals/day & $12(35 \%)$ & $16(28 \%)$ & 0.4701 \\
\hline 4 meals/day & $13(38 \%)$ & $22(39 \%)$ & 0.9727 \\
\hline 5 meals/day & $7(21 \%)$ & $17(30 \%)$ & 0.3334 \\
\hline $\begin{array}{l}\text { KIDMED score } \\
(\mathrm{M} \pm \mathrm{SD}, \text { range) }\end{array}$ & $5.5 \pm 2.47(0-10)$ & $7.14 \pm 2.45(1-11)$ & 0.0028 \\
\hline Optimal adherence $(\geq 8)$ & $8(23 \%)$ & $29(51 \%)$ & 0.01 \\
\hline Medium adherence $(4-7)$ & $20(59 \%)$ & $25(44 \%)$ & 0.1672 \\
\hline Poor adherence $(\leq 3)$ & $6(18 \%)$ & $3(5 \%)$ & 0.05 \\
\hline
\end{tabular}

Statistical differences were evaluated by Student's $t$-test and by chi-squared tests.

As expected, ISS score was significantly higher in active than sedentary adolescents $(p=0.0001)$. Interestingly, in active adolescents a significant higher KIDMED score $(p=0.0028)$ along with a strong increase in the percentage of optimal adherents $(p=0.01)$ and a reduction in poor adherents $(p=0.05)$ to the MD were observed. Moreover, using the results of the KIDMED test, a comparison of the compliance rates for each food was calculated between the two groups and depicted in radar charts which clearly illustrate the gap between the current state (percentage of participants currently adhering to each dietary recommendation) and the ideal situation (100\% compliance) (Figure 1).

There were significant differences between active and sedentary adolescents in the food rates for most of the dietary recommendations. Indeed, active adolescents showed a greater intake of a fruit/day, a second fruit/day, more vegetables/day, nuts $\geq 2$ times/week, and low-fat dairy products for breakfast than sedentary. In the same group of adolescents, the percentages of subjects having breakfast and not consuming sweets or candy every day were significantly higher compared to those of other groups (Figure 1). More interestingly, while we found that ISS and KIDMED scores failed to be associated in sedentary adolescents, they were positively correlated in active adolescents $(r=0.311, p=0.0185)$ (Figure 2).

3.4. Self-Perceived Physical Activity and the Relation to Life Satisfaction in Sedentary and Active Adolescents during the COVID-19 Lockdown

We also evaluated the differences in the items of ISS questionnaire in the two PA groups. As shown in Table 4, a strong increase in active compared to sedentary participants was evident in performing PA at least $1 \mathrm{~h}$ a day at home $(89 \%$ vs. $41 \%, p=0.00001)$ and at least $1 \mathrm{~h} 3$ times a week (93\% vs. 59\%, $p=0.00007)$, as well as resting at least $6-8 \mathrm{~h}$ in the night $(95 \%$ vs. $79 \%, p=0.02)$ and helping in doing some housework $(96 \%$ vs. $85 \%$, $p=0.05)$. 


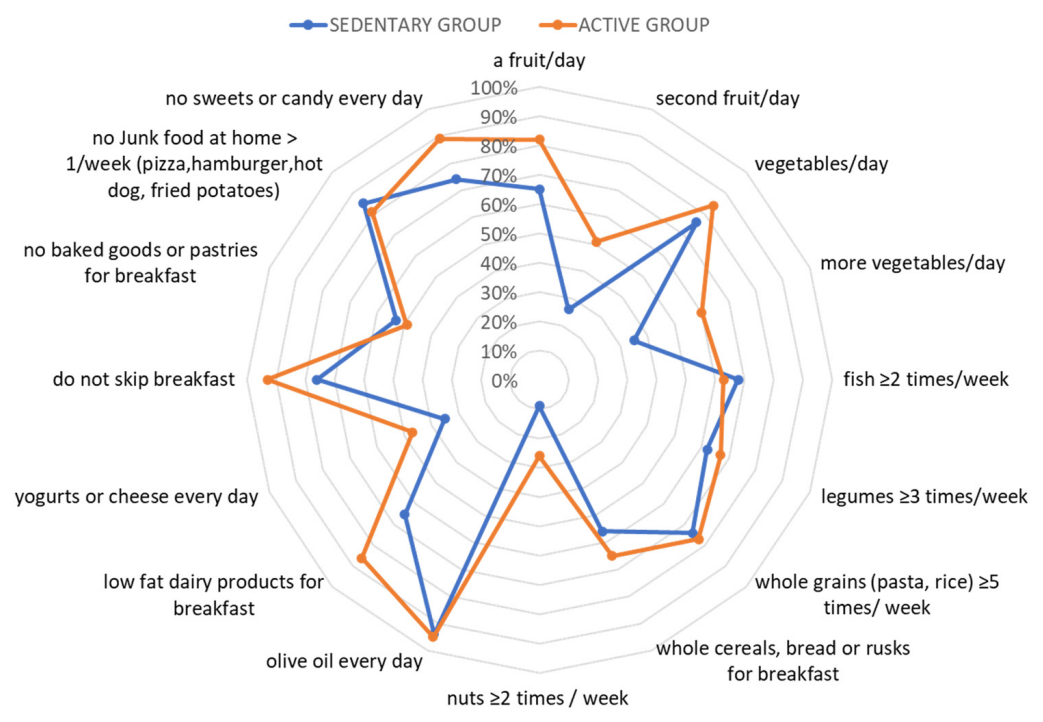

\begin{tabular}{cccc}
\hline KIDMED SCORE & Sedentary & Active & $p$-Value \\
\hline a fruit/day & $65 \%$ & $82 \%$ & 0.05 \\
\hline second fruit/day & $26 \%$ & $51 \%$ & $\mathbf{0 . 0 2}$ \\
\hline vegetables/day & $76 \%$ & $84 \%$ & 0.21 \\
\hline more vegetables/day & $35 \%$ & $60 \%$ & $\mathbf{0 . 0 2}$ \\
\hline fish $\geq 2$ times/week & $68 \%$ & $63 \%$ & 0.66 \\
\hline legumes $\geq 3$ times/week & $62 \%$ & $67 \%$ & 0.63 \\
\hline whole grains (pasta, rice) $\geq 5$ times/week & $74 \%$ & $77 \%$ & 0.69 \\
\hline whole cereals, bread or rusks for breakfast & $56 \%$ & $65 \%$ & 0.49 \\
\hline nuts $\geq 2$ times/week & $9 \%$ & $26 \%$ & $\mathbf{0 . 0 4}$ \\
\hline olive oil every day & $94 \%$ & $95 \%$ & 0.90 \\
\hline low-fat dairy products for breakfast & $65 \%$ & $86 \%$ & $\mathbf{0 . 0 1}$ \\
\hline yogurts or cheese every day & $35 \%$ & $47 \%$ & 0.26 \\
\hline do not skip breakfast & $76 \%$ & $93 \%$ & $\mathbf{0 . 0 2}$ \\
\hline no junk food at home $>1 /$ week (pizza, hamburger, hot dog, fried potatoes) & $83 \%$ & $49 \%$ & 0.72 \\
\hline no sweets or candy every day & $74 \%$ & $81 \%$ & 0.57 \\
\hline
\end{tabular}

Figure 1. Compliance with items from the KIDMED test in active and sedentary adolescents. The radar chart plots the values of each item of the Mediterranean diet score along a separate axis that starts in the center of the chart $(0 \%$ compliance $)$ and ends at the outer ring (100\% compliance). In the table the statistical differences of each item of the Mediterranean diet score between active and sedentary adolescents were evaluated by chi-squared tests.

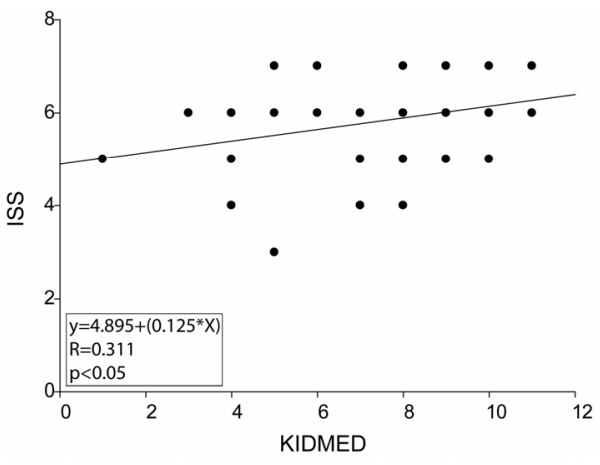

Figure 2. Correlation between ISS score and current lifestyle in active adolescents was analyzed by Pearson's correlation test. For linear regression graph, the linear equation (y), the correlation coefficient (r), and the statistical significance $(p)$ are reported. 
Table 4. Percentages (\%) of sedentary and active adolescents during lockdown with respect of items from the ISS questionnaire.

\begin{tabular}{|c|c|c|c|}
\hline ISS Questionnaire & Sedentary & Active & $p$-Value \\
\hline Are you exercising at least $1 \mathrm{~h}$ a day at home? & $14(41 \%)$ & $51(89 \%)$ & 0.00001 \\
\hline $\begin{array}{l}\text { Currently in your free time, do you spend a maximum of } 2 \mathrm{~h} \text { a day on your } \\
\mathrm{PC} / \text { tablet/TV/smartphone? (excluding the time, you dedicate to studying) }\end{array}$ & $21(62 \%)$ & $44(77 \%)$ & 0.1150 \\
\hline Is physical activity currently performed at home (at least $1 \mathrm{~h}$ ) 3 times a week? & $20(59 \%)$ & $53(93 \%)$ & 0.00007 \\
\hline Having to stay at home all day, do you respect a night's rest of at least $6-8 \mathrm{~h} ?$ & $27(79 \%)$ & $54(95 \%)$ & 0.02 \\
\hline Are you dedicating time to some hobbies? (music, reading, drawing, creative activities) & $28(82 \%)$ & $50(88 \%)$ & 0.4791 \\
\hline $\begin{array}{l}\text { Are you helping the family with some housework? (dusting, making the bed, } \\
\text { setting/clearing the table) }\end{array}$ & $29(85 \%)$ & $55(96 \%)$ & 0.05 \\
\hline Are you using the web to integrate your study activity with other cultural activities? & $18(53 \%)$ & $22(39 \%)$ & 0.1822 \\
\hline
\end{tabular}

Statistical differences were evaluated by chi-squared tests.

Analyzing the PA behaviors into the two groups of active and sedentary adolescents, we asked: "Did you practice physical activity regularly before the COVID-19 emergency or did you gradually start exercising at home?" Table 5 displays results pertaining to reported changes in the PA showing that active adolescents declared to continue physical activity at home in a percentage significantly higher with respect to sedentary ( $75 \%$ vs. $35 \%, p=0.0001$ ). Similarly, sedentary adolescents declared to not practice exercise at home in a greater percentage than in active group ( $41 \%$ vs. $2 \%, p=0.00001)$.

Table 5. Reported changes (\%) in physical activity behavior during the lockdown by sedentary and active adolescents.

\begin{tabular}{|c|c|c|c|}
\hline ITEMS & Sedentary & Active & $p$-Value \\
\hline Yes, I practiced physical activity regularly and I am continuing it at home & $12(35 \%)$ & $43(75 \%)$ & 0.0001 \\
\hline Yes, I practiced physical activity regularly but I'm NOT continuing it at home & $14(41 \%)$ & $1(2 \%)$ & 0.00001 \\
\hline Yes, I gradually started exercising at home & $7(21 \%)$ & $12(21 \%)$ & 0.9579 \\
\hline None of the above & $1(3 \%)$ & $1(2 \%)$ & 0.8833 \\
\hline
\end{tabular}

Statistical differences were evaluated by chi-squared tests.

Finally, significant differences between sedentary and active adolescents with respect of their life satisfaction level related to PA were found (Table 6). Indeed, only a minority of active participants considered it boring to practice PA at home compared to sedentary $(16 \%$ vs. $44 \%, p=0.003)$ and most of them declared to feel good (active: $58 \%$ vs. sedentary: $32 \%, p=0.01)$.

Table 6. Percentages (\%) of sedentary and active adolescents with respect of their consideration of physical activity practiced at home during the lockdown.

\begin{tabular}{cccc}
\hline $\begin{array}{c}\text { In this Period, How Do You Consider Physical } \\
\text { Activity Practiced at Home? }\end{array}$ & Sedentary & Active & $p$-Value \\
\hline Tiring & $3(9 \%)$ & $2(3 \%)$ & 0.2817 \\
\hline Boring & $15(44 \%)$ & $9(16 \%)$ & $\mathbf{0 . 0 0 3}$ \\
\hline It makes me feel good & $11(32 \%)$ & $31(58 \%)$ & $\mathbf{0 . 0 1}$ \\
\hline Statistical differences were evaluated by chi-squared tests. & $5(15 \%)$ & $13(23 \%)$ & 0.3479 \\
\hline
\end{tabular}

\section{Discussion}

In the present web survey, we investigated the self-perceived lifestyle, including PA intensity levels, and eating habits among a cohort of adolescents during the lockdown period caused by the COVID-19 pandemic. Globally, most of the participants (63\% of 
total population) declared an active current lifestyle and no changes with regards of eating habits without gender differences. Interestingly, using the KIDMED questionnaire to assess both the score to the MD and the compliance to the MD recommendations, we observed in all adolescents, as well as in females and in males, an average adherence to the MD. This result confirms our previous investigations on MD adherence conducted in a pre-lockdown period on the same group of adolescents and on a sample of adults living in the same Mediterranean area [12,17]. Similarly, Dragun et al. reported no substantial differences in dietary habits between pre-lockdown and lockdown period, including the overall adherence to the MD and their food choices [18]. In other observations, conflicting data are reported because COVID-19 confinement influenced dietary habits, particularly in younger populations [19]. In fact, data collected by an anonymous online questionnaire on food intake among a group of adolescents from Spain, Italy, Brazil, Colombia, and Chile showed a modified consumption in certain types of foods, such as fried food, sweet food, legumes, vegetables, and fruits which were significantly increased during the lockdown [11]. Moreover, the school closures, coupled with additional socio-behavioral adaptations (e.g., social distancing, quarantining), had a strong impact on the lifestyle activities of children and adolescents across the whole day leading to significant decreases in PA along with increases in sedentary behavior, and disrupted sleep quality in children and adolescents [20]. As is well known, PA is associated with numerous health benefits for adolescents, including cardio-metabolic effects, motor skill development, bone density, and emotional regulation/psychological health [21,22]. Specifically, guidelines and recommendations provided by the WHO for children and adolescents reported that they should participate in moderate-to-vigorous PA at least $1 \mathrm{~h}$ per day mostly aerobic, physical activity, across the week, or they should practice vigorous-intensity aerobic activities at least 3 days a week [23]. Instead, sedentary behaviors indicated often as "screen-based media use behaviors", including watching television (TV), using computers/smartphones, and playing video games are associated with various negative health consequences [24,25]. The adverse consequences resulting from sedentary behaviors include an increased risk of obesity, cardiovascular disease, and all-cause mortality [26]. For that reason, based on guidelines issued by the WHO, the Italian National Institute of Health adapted the recommended levels of PA for different age groups, including in adolescents aged 12-17 years during the COVID-19 outbreak emergency in our country [16,27]. During lockdown, helping to increase the sense of self-efficacy and improve general health of our population, we published a short leaflet and a number of specific suggestions on how to eat well and exercise at home [28]. We have also created an official website of DIMENU project [29] and a Facebook page [30] as innovative ways for assuring additional support and information on health-related issues. Notably, in our study, most adolescents, who declared that their lifestyle was active during the lockdown, have also the highest level of ISS score confirming the self-perceived PA levels. Similarly, as also reported by another recent survey on students during social confinement, it was observed that adolescents tried to carry out PA, getting interested in cooking, reading, and playing board games at home as well [31]. After dividing the population into the active and sedentary groups, we found that active adolescents closely adhered to food- and nutrient-based recommendations. Particularly, we found significant differences in the consumption of some typical Mediterranean foods, such as "a fruit/day", "more vegetables", "a second fruit portion/day", "nuts weekly", "low-fat dairy products for breakfast" as well as in "skipping breakfast" and "no sweets or candy every day". Conflicting data are reported on the association between skipping breakfast and overweight/obesity in adolescents [32]. Thus, further studies are needed to clarify the effects of breakfast on weight management. Our results are in line with other studies in which an increase of consumption of junk food, sweets, and candy were related to the sedentary behavior [9]. In addition, based on our data we highlight the impact of PA on the adherence to the MD and on a subjective well-being and healthy lifestyle in adolescents. In this context, we have recently described that PA associated with a nutrition education program improves the adherence to the MD and reduces systemic inflamma- 
tion in adolescents, suggesting that their combined promotion represent a strategy for assuring the prevention and control of chronic non-communicable diseases over the entire lifespan [33]. Experiences from previous epidemics have also shown that there is a need to maintain optimal nutrition at individual and global levels, in order to positively influence the physical and mental health of the population [34]. In this sense, the knowledge of the dietary and lifestyle habits in each age range is necessary to promote MD patterns and regular PA as a priority public health action after COVID-19 confinement or to develop future reactions to unavoidable pandemics.

This study includes some limitations, such as the small sample size and the online survey. Although a large study population may strengthen the results, in our web survey conducted over a short timeframe a small group of subjects interviewed was sufficient to quickly obtain a picture of adolescent behavior. Regarding the online questionnaire that we used to perform this study, this method does not permit achieving a comparable response quality respect to traditional ones, however, during the lockdown period it represented a unique alternative approach for data collection. We also used a website to promote MD pattern and foster to increase PA intensity levels [29].

\section{Conclusions}

In summary, data from our web survey report that active adolescents have higher adherence to the MD, a subjective well-being and life satisfaction level related to PA respect to sedentary underlining the impact of the PA intensity level on the eating habits and healthy behaviors during the lockdown. These findings suggest the usefulness of planning effective education programs including energy and nutritionally balanced diets as well as regular PA, as a part of wellness programs and of interventions in adolescence for a healthy lifestyle.

Author Contributions: A.G. and G.C. data collection, study design, analysis and interpretation of data and writing the manuscript; E.A., E.M., and S.F. nutritional analyses; C.G. and D.S. analyses and interpretation of data; statistical analyses, D.B. conception of idea, study design, reviewing manuscript. All authors have read and agreed to the published version of the manuscript.

Funding: This work was funded by the EU Regional Operational Programme Calabria, Italy (POR Calabria FESR-FSE 2014-2020) DIMENU (prot. \#52243/2017) and by the Department of Excellence (Italian Law. 232/2016) Department of Pharmacy, Health and Nutritional Sciences, University of Calabria, Italy. The APC was funded by the EU Regional Operational Programme Calabria, Italy (POR Calabria FESR-FSE 2014-2020) DIMENU (prot. \#52243/2017).

Institutional Review Board Statement: The study was conducted according to the guidelines of the Declaration of Helsinki and approved by the Ethic Committee of the University of Calabria, Italy (\#5727/2018).

Informed Consent Statement: Informed consent was obtained from all parents of subjects involved in the study.

Data Availability Statement: The datasets used and/or analyzed during the current study are available from the corresponding author on reasonable request.

Acknowledgments: We sincerely thank all our participants enrolled from the high school "Istituto Istruzione Superiore"-Castrolibero and from sports teams of Cosenza and Paola, located in Calabria Region, Italy. We are thankful to all partners of the DIMENU project (Amphiios S.C.R.L., Istituto Istruzione Superiore-Castrolibero, ELLE 17 S.R.L.). We also thank Francesco Grandinetti for helpful administrative support.

Conflicts of Interest: The authors declare no conflict of interest. 


\section{Appendix A}

Table A1. DiMeNu—Lifestyle survey during the COVID-19 lockdown (DiMeNu-LS).

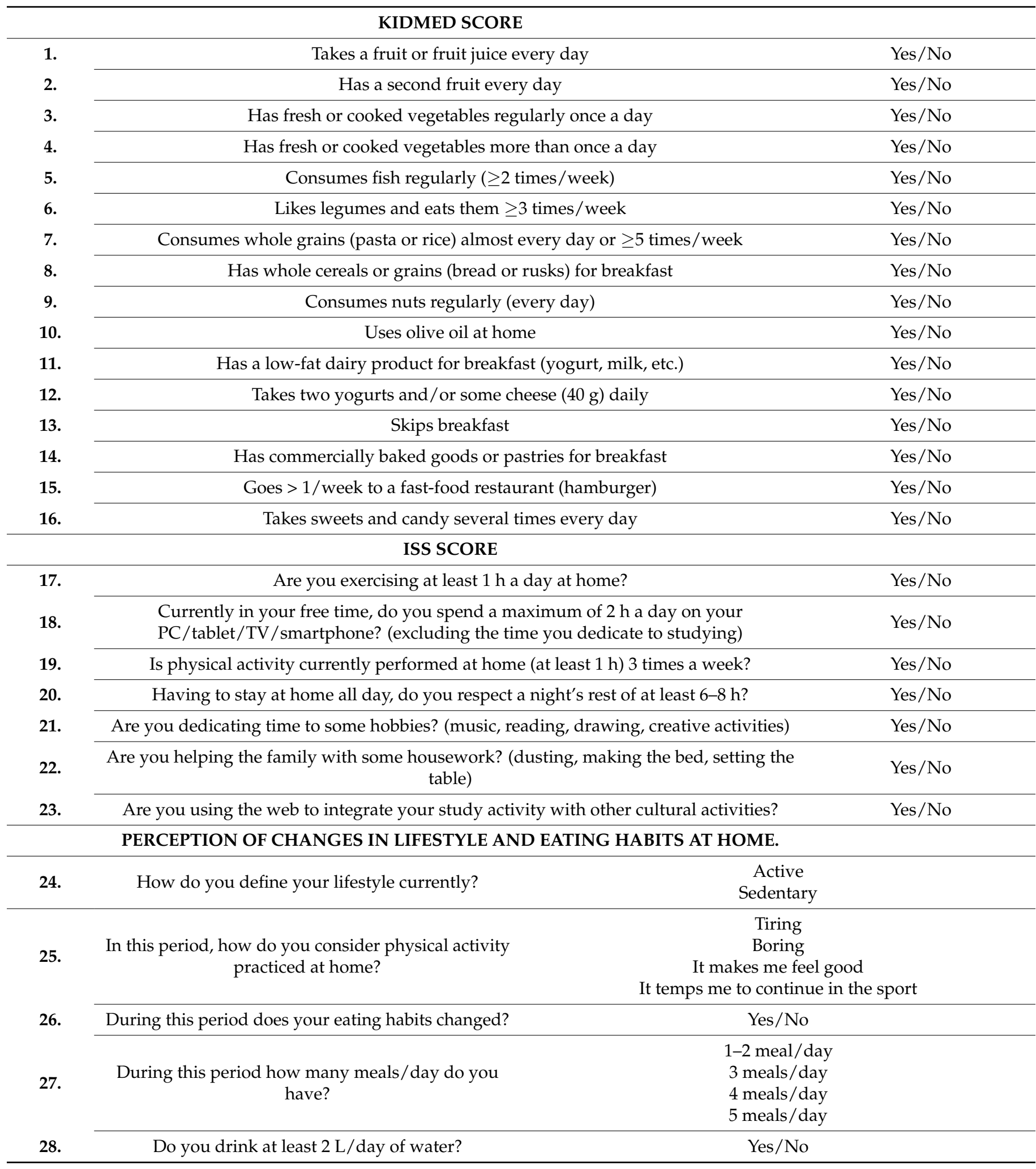


Table A1. Cont.

Before COVID-19 emergency did you practice

29. regularly physical activity or did you gradually start exercising at home?

30. What kind of physical activity are you doing?
Yes, I practiced physical activity regularly and I am continuing it at home

Yes I practiced physical activity regularly but I'm NOT continuing it at home

Yes I gradually started exercising at homeNone of the above

Bodyweight exercises/jumping rope/running or tapis roullant or cyclette/abdominal exercises/step/exercise with objects at home/squat/or lunges or buttocks or legs exercises/push-ups/exergame/stretching/weight training

\section{References}

1. Velavan, T.P.; Meyer, C.G. The COVID-19 epidemic. Trop. Med. Int. Health 2020, 25, 278-280. [CrossRef] [PubMed]

2. Lesser, I.A.; Nienhuis, C.P. The Impact of COVID-19 on Physical Activity Behavior and Well-Being of Canadians. Int. J. Environ. Res. Public Health 2020, 17, 3899. [CrossRef]

3. Government, I. Nuovo Coronavirus. Available online: http://www.salute.gov.it/portale/nuovocoronavirus/dettaglioFaqNuo voCoronavirus.jsp?lingua=italiano\&id=228\#11 (accessed on 7 May 2020).

4. Chen, P.; Mao, L.; Nassis, G.P.; Harmer, P.; Ainsworth, B.E.; Li, F. Coronavirus disease (COVID-19): The need to maintain regular physical activity while taking precautions. J. Sport Health Sci. 2020, 9, 103-104. [CrossRef]

5. Ammar, A.; Brach, M.; Trabelsi, K.; Chtourou, H.; Boukhris, O.; Masmoudi, L.; Bouaziz, B.; Bentlage, E.; How, D.; Ahmed, M.; et al. Effects of COVID-19 Home Confinement on Eating Behaviour and Physical Activity: Results of the ECLB-COVID19 International Online Survey. Nutrients 2020, 12, 1583. [CrossRef] [PubMed]

6. Stockwell, S.T.M.; Tully, M.; Shin, J.; Barnett, Y.; Butler, L.; McDermott, D.; Schuch, F.; Smith, L. Changes in physical activity and sedentary behaviours from before to during the COVID-19 pandemic lockdown: A systematic review. BMJ Open Sport Exerc. Med. 2021, 1, e000960. [CrossRef]

7. Hoffmann, B.; Kobel, S.; Wartha, O.; Kettner, S.; Dreyhaupt, J.; Steinacker, J.M. High sedentary time in children is not only due to screen media use: A cross-sectional study. BMC Pediatr. 2019, 19, 154. [CrossRef]

8. Celis-Morales, C.; Salas-Bravo, C.; Yanez, A.; Castillo, M. Physical inactivity and sedentary lifestyle-The other side of the side effects of the COVID-19 Pandemic. Rev. Med. Chile 2020, 148, 885-886. [CrossRef] [PubMed]

9. Ruiz-Roso, M.B.; de Carvalho Padilha, P.; Matilla-Escalante, D.C.; Brun, P.; Ulloa, N.; Acevedo-Correa, D.; Arantes Ferreira Peres, W.; Martorell, M.; Rangel Bousquet Carrilho, T.; de Oliveira Cardoso, L.; et al. Changes of Physical Activity and Ultra-Processed Food Consumption in Adolescents from Different Countries during Covid-19 Pandemic: An Observational Study. Nutrients 2020, 12, 2289. [CrossRef]

10. Pietrobelli, A.; Pecoraro, L.; Ferruzzi, A.; Heo, M.; Faith, M.; Zoller, T.; Antoniazzi, F.; Piacentini, G.; Fearnbach, S.N.; Heymsfield, S.B. Effects of COVID-19 Lockdown on Lifestyle Behaviors in Children with Obesity Living in Verona, Italy: A Longitudinal Study. Obesity (Silver Spring) 2020, 28, 1382-1385. [CrossRef] [PubMed]

11. Ruiz-Roso, M.B.; de Carvalho Padilha, P.; Mantilla-Escalante, D.C.; Ulloa, N.; Brun, P.; Acevedo-Correa, D.; Arantes Ferreira Peres, W.; Martorell, M.; Aires, M.T.; de Oliveira Cardoso, L.; et al. Covid-19 Confinement and Changes of Adolescent's Dietary Trends in Italy, Spain, Chile, Colombia and Brazil. Nutrients 2020, 12, 1807. [CrossRef]

12. Morelli, C.; Avolio, E.; Galluccio, A.; Caparello, G.; Manes, E.; Ferraro, S.; De Rose, D.; Santoro, M.; Barone, I.; Catalano, S.; et al. Impact of Vigorous-Intensity Physical Activity on Body Composition Parameters, Lipid Profile Markers, and Irisin Levels in Adolescents: A Cross-Sectional Study. Nutrients 2020, 12, 742. [CrossRef]

13. Willett, W.; Rockstrom, J.; Loken, B.; Springmann, M.; Lang, T.; Vermeulen, S.; Garnett, T.; Tilman, D.; DeClerck, F.; Wood, A.; et al. Food in the Anthropocene: The EAT-Lancet Commission on healthy diets from sustainable food systems. Lancet 2019, 393, 447-492. [CrossRef]

14. Garcia Cabrera, S.; Herrera Fernandez, N.; Rodriguez Hernandez, C.; Nissensohn, M.; Roman-Vinas, B.; Serra-Majem, L. Kidmed Test; Prevalence of Low Adherence to the Mediterranean Diet in Children and Young; a Systematic Review. Nutr. Hosp. 2015, 32, 2390-2399. [CrossRef]

15. Revitalizing the Mediterranean Diet from a Healthy Dietary Pattern to a Healthy Mediterranean Sustainable Lifestyle. Available online: http:/ / www.ifmed.org/1st-world-conference-on-the-mediterranean-diet/ (accessed on 30 October 2020).

16. Anche Gli Adolescenti si Muovono a Casa. Available online: https://www.epicentro.iss.it/coronavirus/sars-cov-2-stili-vita-att ivita-fisica-12-17-anni (accessed on 9 February 2021).

17. Caparello, G.; Galluccio, A.; Giordano, C.; Lofaro, D.; Barone, I.; Morelli, C.; Sisci, D.; Catalano, S.; Ando, S.; Bonofiglio, D. Adherence to the Mediterranean diet pattern among university staff: A cross-sectional web-based epidemiological study in Southern Italy. Int. J. Food Sci. Nutr. 2020, 71, 581-592. [CrossRef]

18. Dragun, R.; Vecek, N.N.; Marendic, M.; Pribisalic, A.; Divic, G.; Cena, H.; Polasek, O.; Kolcic, I. Have Lifestyle Habits and Psychological Well-Being Changed among Adolescents and Medical Students Due to COVID-19 Lockdown in Croatia? Nutrients 2020, 13, 97. [CrossRef] [PubMed] 
19. Glabska, D.; Skolmowska, D.; Guzek, D. Population-Based Study of the Changes in the Food Choice Determinants of Secondary School Students: Polish Adolescents' COVID-19 Experience (PLACE-19) Study. Nutrients 2020, 12, 2640. [CrossRef] [PubMed]

20. Bates, L.C.; Zieff, G.; Stanford, K.; Moore, J.B.; Kerr, Z.Y.; Hanson, E.D.; Barone Gibbs, B.; Kline, C.E.; Stoner, L. COVID-19 Impact on Behaviors across the 24-Hour Day in Children and Adolescents: Physical Activity, Sedentary Behavior, and Sleep. Children 2020, 7, 138. [CrossRef] [PubMed]

21. Janssen, I.; Leblanc, A.G. Systematic review of the health benefits of physical activity and fitness in school-aged children and youth. Int. J. Behav. Nutr. Phys. Act. 2010, 7, 40. [CrossRef]

22. Saunders, T.J.; Gray, C.E.; Poitras, V.J.; Chaput, J.P.; Janssen, I.; Katzmarzyk, P.T.; Olds, T.; Connor Gorber, S.; Kho, M.E.; Sampson, M.; et al. Combinations of physical activity, sedentary behaviour and sleep: Relationships with health indicators in school-aged children and youth. Appl. Physiol. Nutr. Metab. 2016, 41, S283-S293. [CrossRef]

23. WHO. Physical Activity. Available online: https://www.who.int/news-room/fact-sheets/detail/physical-activity\#: \{\}:text=Chi ldren \%20and\%20adolescents\%20aged\%205,least\%203\%20days\%20a\%20week (accessed on 9 February 2021).

24. Iannotti, R.J.; Kogan, M.D.; Janssen, I.; Boyce, W.F. Patterns of adolescent physical activity, screen-based media use, and positive and negative health indicators in the U.S. and Canada. J. Adolesc. Health 2009, 44, 493-499. [CrossRef]

25. Leung, M.M.; Agaronov, A.; Grytsenko, K.; Yeh, M.C. Intervening to Reduce Sedentary Behaviors and Childhood Obesity among School-Age Youth: A Systematic Review of Randomized Trials. J. Obes. 2012, 2012, 685430. [CrossRef] [PubMed]

26. Wu, X.Y.; Han, L.H.; Zhang, J.H.; Luo, S.; Hu, J.W.; Sun, K. The influence of physical activity, sedentary behavior on health-related quality of life among the general population of children and adolescents: A systematic review. PLoS ONE 2017, 12, e0187668. [CrossRef] [PubMed]

27. ISS. Teenagers Can also Take Exercise at Home. Available online: https://www.epicentro.iss.it/en/coronavirus/pdf/physical-a ctivity-at-home-for-adolescents-12-17-yy.pdf (accessed on 9 February 2021).

28. UNICAL. Coronavirus, I Consigli Degli Esperti per uno Stile di Vita Salutare. Available online: https://www.unical.it/portale/ portaltemplates/view/view.cfm?98612 (accessed on 9 February 2021).

29. Dieta Mediterranea e Nuoto. Available online: https:/ / www.dimenu.it/ (accessed on 9 February 2021).

30. Dieta Mediterranea e Nuoto Facebook Page. Available online: https:/ / www.facebook.com/dimenu2019 (accessed on 9 February 2021).

31. Pigaiani, Y.; Zoccante, L.; Zocca, A.; Arzenton, A.; Menegolli, M.; Fadel, S.; Ruggeri, M.; Colizzi, M. Adolescent Lifestyle Behaviors, Coping Strategies and Subjective Wellbeing during the COVID-19 Pandemic: An Online Student Survey. Healthcare $2020,8,472$. [CrossRef] [PubMed]

32. Ardeshirlarijani, E.; Namazi, N.; Jabbari, M.; Zeinali, M.; Gerami, H.; Jalili, R.B.; Larijani, B.; Azadbakht, L. The link between breakfast skipping and overweigh/obesity in children and adolescents: A meta-analysis of observational studies. J. Diabetes Metab. Disord. 2019, 18, 657-664. [CrossRef] [PubMed]

33. Morelli, C.; Avolio, E.G.; Caparello, G.; Manes, E.; Ferraro, S.; Caruso, A.; De Rose, D.; Barone, I.; Adornetto, C. Nutrition Education Program and Physical Activity affect the Adherence to the Mediterranean Diet and associate with lower Inflammatory Biomarker levels in Healthy Adolescents: The DIMENU longitudinal study. Front. Nutr. 2021. under review.

34. Naja, F.; Hamadeh, R. Nutrition amid the COVID-19 pandemic: A multi-level framework for action. Eur. J. Clin. Nutr. 2020, 74, 1117-1121. [CrossRef] [PubMed] 